\title{
Association between Self-Efficacy and Health Behaviour in Disease Control: A Systematic Review
}

\author{
Affendi $\mathrm{I}^{1}$, Nor Asiah Muhamad ${ }^{2}$, Normi $\mathrm{M}^{3}$, Mohd Hatta $\mathrm{AM}^{2}$, Noor Aliza L ${ }^{2}$, \\ Md Sabtuah $\mathrm{MR}^{4}$, Mohd Farid B ${ }^{5}$, Zanariah $\mathrm{Z}^{1}$ \& Suraiya $\mathrm{SM}^{1}$ \\ ${ }^{1}$ Health Education Division, Ministry of Health Malaysia, Block E10, Complex E, Federal Government \\ Administration Centre, 62590, Putrajaya, Malaysia \\ ${ }^{2}$ Institute for Public Health, Ministry of Health Malaysia, Malaysia \\ ${ }^{3}$ Faculty of Science \& Technology, Open University Malaysia, Malaysia \\ ${ }^{4}$ Institute for Behavioural Research, Ministry of Health, Malaysia \\ ${ }^{5}$ Institute for Medical Research, Ministry of Health Malaysia, Malaysia \\ Correspondence: Nor Asiah Muhamad, Center for Communicable Disease Research, Institute for Public Health, \\ Jalan Bangsar, Kuala Lumpur 50590, Malaysia. Tel: 60-123-390-723. E-mail: norasiahdr@gmail.com
}

\author{
Received: October 9, 2017 Accepted: November 5, 2017 Online Published: November 20, 2017 \\ doi:10.5539/gjhs.v10n1p18 URL: https://doi.org/10.5539/gjhs.v10n1p18
}

\begin{abstract}
Background: Self-efficacy is defined as his or her belief of their capacity to produce specific performance attainments where represent the ability of positive and negative control over their own motivation, behavior, and social environment. Interventions to increase self-efficacy are a potentially effective way of changing health behavior towards attractive results, however the subject has not been systematically reviewed. This review aims to assess the relationship between self-efficacy and change in good health behaviour.
\end{abstract}

Methods: A comprehensive and extensive search of four bibliographic data bases was conducted for papers reporting health promotion and educational interventions that explicitly targeted self-efficacy in order to change health behaviour in achieving better disease control.

Results: Out of 314 studies, 13 were included in the review. Of these, 8 were found to have significant association between self-efficacy and specific health behaviours. However, 5 studies failed to show any significant prediction towards health behavior. Another 6 studies showed either significant mediation effect or indirect relationship of self-efficacy and health behaviours.

Conclusions: Self-efficacy appears to be an important psychosocial construct that may directly or indirectly affect health behaviour to control diseases. Self-efficacy may also function as a link between effective health promotion and educational interventions and health behaviour change in disease control.

Keywords: self-efficacy, health behaviour change, health education intervention, health promotion intervention, mediation effect

\section{Introduction}

The main aim of health promotion and educational interventions is to increase knowledge, attitudes and change behaviours to control or prevent diseases. According to health promotion interventions, knowledge and positive attitudes usually increase (Brega et al., 2013; Ezedinachi et al., 2002; Pai, Hong, \& Hsu, 2006). However, sustained behaviour change is harder to achieve (Schwarzer, 2008). Knowledge alone may not always be a good predictor of improved health behaviour. The relationship between knowledge, attitude and behaviour is complex and difficult to establish (Chaffee \& Roser, 1986). In order to understand this complex relationship, there are multiple theoretical perspectives that have been used to develop effective health promotion and educational interventions. Self-efficacy is seen as one such relevant psychological variable that connects the impact of health promotion and educational intervention on knowledge and attitude to health behaviour enactment (Albert Bandura \& Wood, 1989; Bouffard-Bouchard, 1990; Strecher, 1983). 


\subsection{Self-Efficacy}

Self-efficacy is defined as people's belief in their capabilities to achieve different levels of performance attainment (Bouffard-Bouchard, 1990). It is a key construct within several theories that are popular within health psychology such as Social Cognitive Theory (Albert Bandura, 1997), Protection Motivation Theory (Rogers, 1975), Transtheoretical Model (Prochaska \& DiClemente, 1982), Health Action Process Approach (Schwarzer, 2014) and Theory of Planned Behaviour (A Bandura, 1977; Albert Bandura, 1978). According to Bandura in his Social Cognitive Theory, self-efficacy has an influence on preparing for action because self-efficacy levels can enhance or impede motivation to behaviour change, for instance 'I intend to reduce my smoking habit' or 'I intend eat fruit and vegetables regularly' (Maibach, Flora, \& Nass, 1991). Apart from involving motivation, self-efficacy is also directly related to behaviour enactment, referring to the confidence that one can employ the skills necessary to resist temptation, cope with stress and mobilize one's resources required to meet situational demands (Schwarzer \& Chung, 1996).

\subsection{Mediator of Health Determinants}

A large body of related research supports the predictive value that self-efficacy has on health behaviour change. Self-efficacy either has significant direct effect on behaviours (Baldwin et al., 2006; Hendriksen, Pettifor, Lee, Coates, \& Rees, 2007; Oei \& Burrow, 2000; Scholz, Sniehotta, \& Schwarzer, 2005; Strauss, Rodzilsky, Burack, \& Colin, 2001) or it becomes a mediator between other psychological variables to health behaviours (Darker, French, Eves, \& Sniehotta, 2010; Motl et al., 2002). Although self-efficacy is an important determinant of a number of health behaviours, it is unclear of how to change this psychological construct as to give impact on behaviours as a result of health promotion and educational intervention (Ashford, Edmunds, \& French, 2010). Currently, most reviews that were conducted usually involved interventions without health promotion methods and activities on effectiveness of self-efficacy as a mediator to physical activity or substance abuse and HIV prevention (Baranowski, Anderson, \& Carmack, 1998; Hyde, Hankins, Deale, \& Marteau, 2008; Lewis, Marcus, Pate, \& Dunn, 2002; Marcus, King, Clark, Pinto, \& Bock, 1996; Mize, Robinson, Bockting, \& Scheltema, 2002; Sherwood \& Jeffery, 2000). Hence, this review was carried out to identify interventions that applied health promotion and education activities as a tool to enhance self-efficacy for behavioural change in control and prevention of any related diseases.

\section{Methodology}

\subsection{Search Strategy}

A comprehensive search strategy was developed to search all published articles that can be considered eligible for inclusion in this review. The search strategy was adapted from PubMed for use in the electronic databases. The literature search identified potential studies in all languages. Non-English language papers were translated and assessed for potential inclusion in the review as necessary.The following electronic databases were searched to identify potential studies:

- MEDLINE (1948- March 2017)

- EMBASE (1974- March 2017)

- Pro Quest (PsycInfo) (1977- March 2017)

- Web of Science (1966- March 2017)

Full details of the search strings are given in Table 1. No restrictions were made regarding publication year. Two authors independently screened titles and abstract for inclusion in the review. Any disagreement was resolved by discussion between all authors. The screening process yielded 52 potentially relevant articles. Full text articles were retrieved and independently assessed by two authors. All disagreements were referred to third author. 
Table 1. Search String

Search Strategy for Ovid SP MEDLINE and EMBASE, with suffix.mp (mp=ti, ab, sh, hw, tn, ot, dm, mf, dv, kw, ps, rs, nm, ui) Self-efficacy terms: Self-Efficacy OR Efficacy OR Self- Esteem OR Esteem OR Self-Efficacy of Social Cognitive Theory OR Self-Efficacy of Health Belief Model OR Self-efficacy of Health Psychology

AND

Behavioural Change terms: Behaviour Change OR Change in Behaviour OR Health Behaviour Change OR Practices OR Behavioural Actions

AND

Health Promotion Intervention Terms: Health Promotion Intervention OR Health Education OR Health Campaigns OR Behavioural Intervention OR Community Intervention OR Educational Intervention OR Intervention

Not limited to language.

\subsection{Study Selection and Eligibility Criteria}

A title and abstract review of these studies were conducted based on inclusion and exclusion criteria developed by the authors.

\subsubsection{Inclusion Criteria}

Participants for the included studies were not limited to specific characteristic such age or educational status. Only studies that used health promotion and education as intervention to prevent any diseases and measure the association between self-efficacy and behaviour change with quantitative outcomes were included in this review.

\subsubsection{Exclusion Criteria}

All studies based on laboratory or semi-field settings and qualitative analysis were excluded. Studies that focused on general health were also excluded in this review.

\subsection{Validity Assessment}

The risk of bias for included studies were assessed using 4 domains namely selection of participants, measurement of self-efficacy, analysis method used in assessing relationship between self-efficacy and health behaviour and finally attrition bias particularly loss to follow-up, missing data, or incomplete reporting (Table 2).

Table 2. Validity Assessment and Limitations

\begin{tabular}{|c|c|c|c|}
\hline Study & Lost to Follow-up & Statistical Test and Adjustment & Limitations \\
\hline $\begin{array}{l}\text { Therawiwat } \\
\text { M. et. al, } \\
2005 \\
\text { Thailand }\end{array}$ & Not mentioned & $\begin{array}{l}\text { Multivariate analysis } \\
\text { Key stakeholders, representative } \\
\text { household members, factors }\end{array}$ & Generalizability \\
\hline $\begin{array}{l}\text { Morisky D.E. } \\
\text { et. al, } 2001\end{array}$ & $\begin{array}{l}79 \% \text { agreed to participate } \\
\text { The overall rate of treatment } \\
\text { completion was } 79.8 \%(84.8 \% \\
\text { for combined intervention } \\
\text { group, } 80.3 \% \text { in the peer } \\
\text { counselling group, } 77.8 \% \\
\text { receiving usual care and } \\
76.4 \% \text { in the incentive group). }\end{array}$ & $\begin{array}{l}\text { Multivariate analysis } \\
\text { Race/ethnicity, age, sex, } \\
\text { education }\end{array}$ & $\begin{array}{l}\text { Effect of the interviews on } \\
\text { participants; monetary incentives } \\
\text { may influence behaviour toward } \\
\text { treatment adherence. } \\
\text { Questionnaires that was translated } \\
\text { from English to Spanish, scales of } \\
\text { strongly agree to strongly disagree } \\
\text { did not have exact equivalents in } \\
\text { Spanish; so the validity of those data } \\
\text { may have been compromised. }\end{array}$ \\
\hline $\begin{array}{l}\text { Luszczynska } \\
\text { A., } 2004\end{array}$ & $\begin{array}{l}61 \% \quad \text { longitudinal data } \\
\text { collected during } 2 \text { waves were } \\
\text { available. } 58 \% \quad \text { samples } \\
\text { remained after listwise } \\
\text { deletion of mission data. }\end{array}$ & $\begin{array}{l}\text { Intervention and control group } \\
\text { were compared using t-test and } \\
\text { structural equation modeling } \\
\text { using AMOS. } \\
\text { No adjustment }\end{array}$ & $\begin{array}{l}\text { Relies on self-reported measures; no } \\
\text { objective or observational indicators } \\
\text { of BSE performance. Retrospective } \\
\text { self-reports dealing with longer } \\
\text { periods of time can be biased. }\end{array}$ \\
\hline
\end{tabular}




$\begin{array}{lll}\begin{array}{l}\text { Piaseu N, et. } \\ \text { al, } 2010\end{array} & \begin{array}{l}100 \text { women participated from } \\ 140 \text { recruited. }\end{array} & \begin{array}{l}\text { Multivariate analysis and } \\ \text { mediation analysis }\end{array} \\ \text { Thailand } & 10 \text { participant did not } & \text { Exercise and calcium behaviours } \\ & \text { completely fill out the } \\ & \text { instruments and dropped out } \\ & \text { from study }\end{array}$

$\begin{array}{lll}\text { Berendes } \mathrm{S} \& & 881 \text { samples in study } 2 \text { as } \\ \text { Rimal RN, } & \begin{array}{l}\text { compared to } 891 \text { samples in } \\ \text { study } 1\end{array}\end{array}$

Cameroon

Burke LE, et. 333 patients screened, 49\% al., 2005 (163) were eligible. 79 expressed interest to USA participate, 65 consented to participate.

$68 \%$ response to follow up questionnaires after 2 months of study completion.

$\begin{aligned} & \text { Iever-Landis } \\ & \mathrm{CE}, \text { et. al, } 436 \text { participants, } 42 \text { were } \\ & 2003\end{aligned}$
$\begin{aligned} & \text { not eligible and } 40 \text { were } \\ & \text { dropped from the data } \\ & \text { analyses due to missing data. }\end{aligned}$

USA

Burke V., et. Of 118 participants in control al., 2008 group 98 completed the 4-month intervention and 90 completed follow-up

Australia

$\begin{array}{lrr}\text { Of } 123 \text { participants in } \\ \text { intervention } & \text { group, } 106 \\ \text { completed the } & \text { 4-month } \\ \text { intervention and } & 102 \\ \text { completed follow-up. } & \end{array}$

\begin{tabular}{l|l|}
$\begin{array}{l}\text { Strathdee SA., } \\
\text { et. al, } 2009\end{array}$ & $\begin{array}{l}\text { Of } 924 \text { participants, } 409 \\
\text { included in the study because } \\
\text { the study aimed to determine } \\
\text { within-group factors. }\end{array}$ \\
\hline
\end{tabular}

\section{Multivariate analysis using} STATA

Knowledge, age, gender, marital status, stigma, self-efficacy and education

Intervention and control group

were compared using bivariate analysis

No adjustment

Multivariate analysis and SEM using AMOS

Self-efficacy, knowledge
Sex, time spent for physical activity, stage of change
Multivariate analysis

Drugs injection

Sample was homogeneous and small; limits generalizability.

Self-reported exercise measures were not validated with objective measures.

Questionnaires translation and back translation might be misleading.

Household surveys using face-to-face interviews may bias; as a result of social desirability, led to under-reporting of stigma and over-reporting of willingness to test for HIV

\section{Absence of follow up}

Small sample; limits generalizability.
Sampling; Girl Scouts may be already related to a healthy eating; led to over report of Calcium intake.

Sample primarily Caucasian, limits the generalizability.

Weak path coefficients that were included in structural model may influence predictors result in eating behaviour.

Multivariate analysis, moderating analysis

Sample all Caucasian, limits the generalizability.
Follow up period was relatively short; intervention effects may have waned over time.

Low power to detect significant interaction

Relied on self-report

Sample was geographically limited; limit generalizability; studied only 2 Mexico-USA border cities. 


\begin{tabular}{ll}
\hline Critchley & Retention rate $89 \% ; 12 \%$ in \\
$\mathrm{CR}$, et. al, & intervention and $8 \%$ in control \\
2012 & $\begin{array}{l}\text { group failed to complete the } 2 \\
\text { measurements. }\end{array}$
\end{tabular}

Australia
Sharma Not mentioned. Study of SV., et. al., secondary data analysis. 2010

USA

Chariyeva Of 183 participants, $85 \%$ were
Z., et. al, retained at 4-month follow-up,
2011
$\begin{aligned} & 78 \% \text { at } 8 \text { month and } 66 \% \text { at the } \\ & 12 \text { month follow-up. }\end{aligned}$

USA
Multivariate analysis and

mediation analysis

Knowledge, positive mood, motivation, exercises self-efficacy and diet self-efficacy).

Mediating and moderating analysis

No adjustment

Of 681 in control, only $37 \%$ me the inclusion criteria. Of those $85 \%$ completed $2^{\text {nd }}$ assessment, $71 \%$ third, $62 \%$ forth and $61 \%$ completed fifth assessment.

Of 432 patients in intervention group, 151 included in the study. 15 patients did not complete the intervention. Of 132 patients remained, $97 \%$ completed $2^{\text {nd }}$ assessment, $90 \%$ third, $76 \%$ forth and $77 \%$ completed fifth assessment.

\section{Multivariate analysis, mediation} analysis

Age, educational attainment, having a main partner, sexual identity, date of HIV diagnosis, having an undetectable HIV viral load, clinic site, counsellor, proportion of counselling sessions done by telephone, history of previous enrolment in a study providing MI counselling, binge drinking in the last 3 months and cocaine/crack use in the last 3 months.
Unequal $(2: 1)$ of genders in control and intervention group.

Relied on self-report of measurement of exercise behaviour.

In both structural model, relatively weak direct influences on the exercise behaviours, with path coefficients ranging from -0.04 to 0.23 .

\section{Relied on secondary data \\ Cross-sectional study, causality cannot be determined.}

Ethic homogeneity of the sample; limit generalizability.

No covariates were not adjusted for in the analysis

Self-reported data rather than observational.

Absence of randomization of counselling time; limit assessment on causal relationship between amount of motivational interviewing received and sexual behaviour.

On-going health promotion campaign during survey; influence participants to practice safer sex.

The study site was not randomly selected; threats to external validity and limit generalizability.

\subsection{Data extraction}

Relevant papers were retrieved and automatically entered into reference software (Endnote version 17). Data were extracted using a standardized data extraction form developed by the authors. Two review authors extracted study characteristics from included studies which include information on study design, total duration of study, study location, study setting and study period. Information on participants were also collected regarding total number of participants, mean age or age range and gender. For interventions, all information on description of intervention, duration and content of intervention were collected. All out comes were described as effectiveness of self-efficacy and its mediating effect on health behaviour. 


\section{Results}

The search identified 314 citations of which 13 papers were included in the analysis. Figure 1 shows the flow diagram detailing the search process and inclusion of studies in this review. Due to significant heterogeneity of study designs, settings, participants, diseases, type of health promotion and education methods or activities and outcome measures, the key study outcomes were synthesized narratively according to health behaviour to control communicable and non-communicable public health diseases. Details of each study and overall validity across the entire set of included studies are broadly summarized in Tables 2 and 3.

\subsection{Characteristics of Included Studies}

Out of 13 papers included in this review, 5 papers are based in the United States of America, 2 in the Europe, 2 in South East Asia, 2 in Australia and the remainder 2 papers are from Africa and South America. Sample size ranged from 65 to 1113 and participants' mean age ranged from 9.37 to 62.5 years (Table 3).

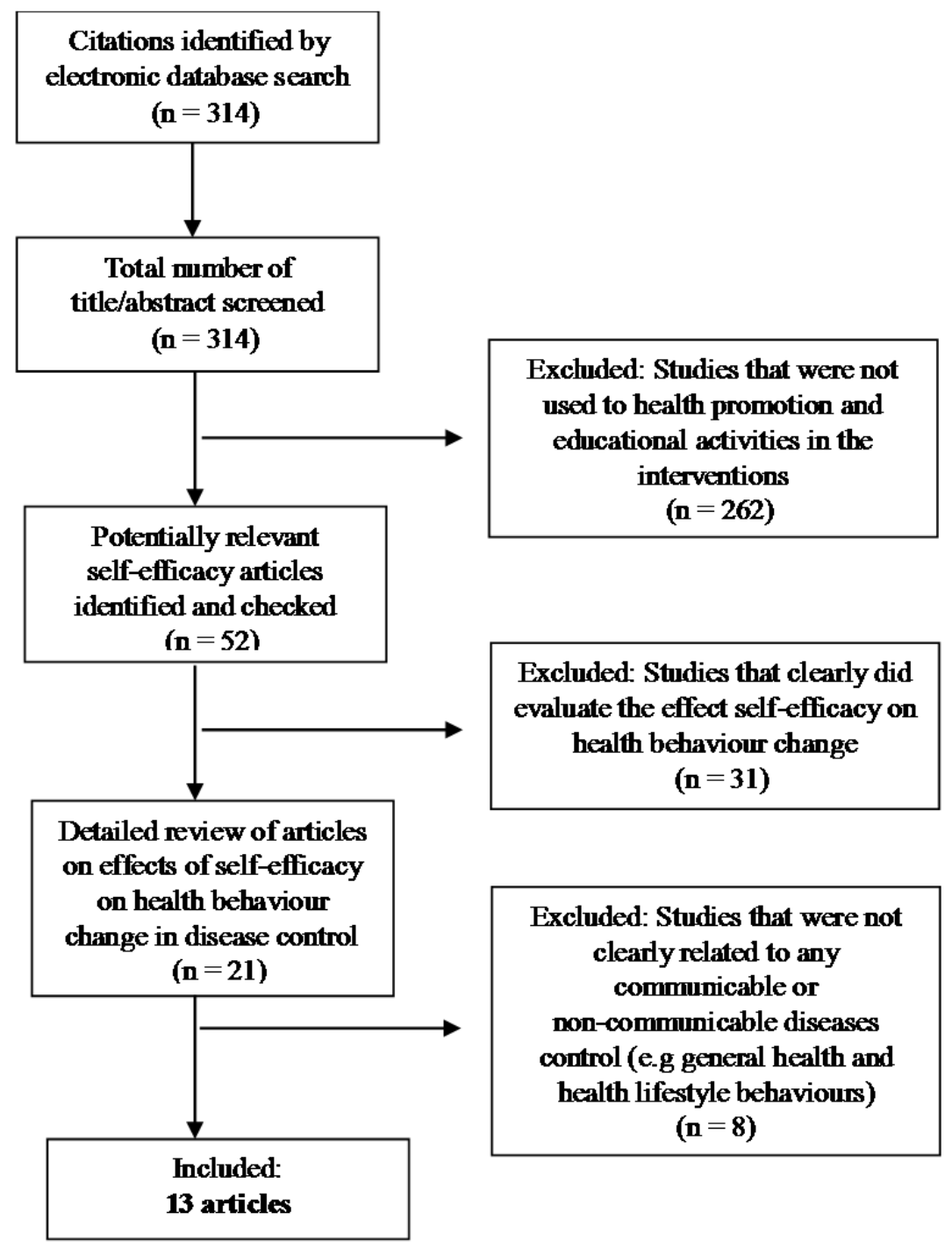

Figure 1. Search process and study selection 
Table 3. Study Characteristics and Outcomes

\begin{tabular}{|c|c|c|c|c|c|c|c|}
\hline Study & Type of behaviors & Diseases & $\begin{array}{l}\text { Design\& Health Promotion } \\
\text { Intervention }\end{array}$ & $\begin{array}{l}\text { Main outcome } \\
\text { measure }\end{array}$ & $\begin{array}{l}\text { Time } \\
\text { between } \\
\text { plan and } \\
\text { outcome } \\
\text { measure }\end{array}$ & Sample & Results \\
\hline $\begin{array}{l}\text { Therawiwat } \\
\text { M. et. al, } \\
2005 \\
\text { Thailand }\end{array}$ & $\begin{array}{l}\text { Regular larval survey } \\
\text { practices }\end{array}$ & Dengue fever & $\begin{array}{l}\text { Experimental (Controlled trial } \\
\text { with } 2 \text { arms) } \\
\text { Some treatment control group } \\
\text { Community-based (Training } \\
\text { sessions, group discussions, } \\
\text { dialogue and monthly meeting } \\
\text { with the key stakeholders, } \\
\text { experience sharing) }\end{array}$ & $\begin{array}{l}\text { Interview } \\
\text { questionnaires and } \\
\text { larval survey form } \\
\text { (Container Index, } \\
\text { House Index \& } \\
\text { Breteau Index) }\end{array}$ & 1 year & $\begin{array}{l}287 \text { ( } 53 \text { key community } \\
\text { leaders and } 234 \\
\text { representatives of household } \\
\text { members) } \\
55.4 \% \text { Female } \\
53.7 \% \text { age } 30-49 \text { years old }\end{array}$ & $\begin{array}{l}\text { Self-efficacy did not significantly } \\
\text { predict the larval survey practices } \\
\text { ( } \mathrm{F}=0.312, p=0.732 \text { ) (Multiple } \\
\text { Classification Analysis). } \\
\text { However, self-efficacy was more } \\
\text { reliable in explaining larval } \\
\text { survey practice }-70 \% \text { of the } \\
\text { samples having high levels of } \\
\text { self-efficacy did regular larval } \\
\text { surveys. (Descriptive analysis). }\end{array}$ \\
\hline $\begin{array}{l}\text { Morisky D.E. } \\
\text { et. al, } 2001 \\
\text { USA }\end{array}$ & $\begin{array}{l}\text { Consistent } \\
\text { medication-taking and } \\
\text { complete care }\end{array}$ & Tuberculosis & $\begin{array}{l}\text { Experimental (Cluster randomised } \\
\text { trial with } 4 \text { arms 3I and 1C) } \\
\text { Some treatment control group } \\
\text { Peer counselling and behavioural } \\
\text { contract (parent-participant } \\
\text { contingency contract } \\
\text { intervention). }\end{array}$ & Questionnaires & 6 months & $\begin{array}{l}794 \text { adolescents who received } \\
\text { treatment of latent } \mathrm{TB} \\
\text { Mean age }=15.2(\mathrm{SD}=1.9) \\
51 \% \text { Females } \\
77.8 \% \text { Hispanic Americans }\end{array}$ & $\begin{array}{l}\text { Self-efficacy has significant } \\
\text { relationship with } \\
\text { medication-taking behaviour } \\
\text { after the post test }(\mathrm{R}=0.254, \\
p=0.002) \text {. } \\
\text { Self-efficacy was strongly } \\
\text { correlated with the completion of } \\
\text { care ( } \mathrm{R}=0.367, p=0.002) \text {. }\end{array}$ \\
\hline $\begin{array}{l}\text { Luszczynska } \\
\text { A., } 2004 \\
\text { Poland }\end{array}$ & $\begin{array}{l}\text { Breast } \\
\text { self-examination }\end{array}$ & Breast Cancer & $\begin{array}{l}\text { Experiment (Cluster randomised } \\
\text { trial with } 2 \text { arms) } \\
\text { Some treatment control group } \\
\text { Classes, breast silicone model for } \\
\text { lump detection (modelling) and } \\
\text { printed materials }\end{array}$ & Questionnaires & 15 weeks & $\begin{array}{l}720 \text { female students } \\
\begin{array}{l}\text { During two waves longitudinal } \\
\text { data collection ( } 440 \text { women) }\end{array} \\
\text { A final sample ( } 417 \\
\text { participants) }- \text { List wise } \\
\text { deletion of missing data } \\
\text { Mean age }=23.20(\mathrm{SD}=5.61)\end{array}$ & $\begin{array}{l}\text { Results from Structural Equation } \\
\text { Model: } \\
\text { Maintenance self-efficacy } \\
\text { predicted change in } \mathrm{BSE} \\
\text { performance }(\mathrm{B}=0.19, p<0.05) \text { in } \\
\text { intervention group. } \\
\text { Preaction self-efficacy was only } \\
\text { significant in predicting intention } \\
\text { for BSE in both groups, } \\
\text { intervention }(\mathrm{B}=0.44, p<0.05) \\
\text { and control }(0.21, p<0.05) \text {. }\end{array}$ \\
\hline
\end{tabular}




\begin{tabular}{|c|c|c|c|c|c|c|c|}
\hline $\begin{array}{l}\text { Piaseu N, et. } \\
\text { al, } 2010 \\
\text { Thailand }\end{array}$ & $\begin{array}{l}\text { Health eating (calcium } \\
\text { intake) } \\
\text { Exercise }\end{array}$ & Osteoporosis & $\begin{array}{l}\text { Experiment (Cluster randomised } \\
\text { trial with } 2 \text { arms) } \\
\text { No treatment control group } \\
\text { Osteoporosis educational program } \\
\text { (3-hour courses including } \\
\text { instructional materials, slide } \\
\text { presentation, informational } \\
\text { materials and demonstration) }\end{array}$ & $\begin{array}{l}\text { Questionnaires of } \\
\text { Osteoporosis } \\
\text { Knowledge Test } \\
\text { (OKT) } \\
\text { Questionnaires of } \\
\text { Osteoporosis Health } \\
\text { Belief Model Scale } \\
\text { (OHBS) } \\
\text { Questionnaires of } \\
\text { Osteoporosis } \\
\text { self-efficacy scale } \\
\text { (OSES) } \\
\text { 24-hour dietary recall } \\
\text { for calcium intake } \\
\text { behaviour }\end{array}$ & 2 weeks & $\begin{array}{l}100 \text { women enrolled in the } \\
\text { first year nursing program } \\
50 \text { in experiment group, and } \\
50 \text { in control group } \\
\text { Mean age }=18.5(\mathrm{SD}=0.6)\end{array}$ & $\begin{array}{l}\text { After intervention, self-efficacy } \\
\text { directly predicted calcium intake } \\
\text { behaviour }(\mathrm{B}=0.38, p<0.05) \text { and } \\
\text { exercise ( } \mathrm{B}=0.36, p<0.01) . \\
\text { Self-efficacy was a mediator } \\
\text { between attitudes and calcium } \\
\text { intake behaviour ( } \mathrm{B}=0.32 \text {, } \\
p<0.05 \text { ) and knowledge and } \\
\text { exercise }(\mathrm{B}=0.31, p<0.001) .\end{array}$ \\
\hline $\begin{array}{l}\text { Berendes S } \\
\text { \&Rimal RN, } \\
2011 \\
\text { Cameroon }\end{array}$ & HIV testing uptake & HIV / AIDS & $\begin{array}{l}\text { Observational (Cross-sectional } \\
\text { study based on previous } \\
\text { intervention) } \\
\text { One-off surveys based on previous } \\
\text { health promotion program } \\
\text { Radio program, posters, } \\
\text { pamphlets, brochures, factsheets, } \\
\text { community events, drama, } \\
\text { billboards, wall stickers, } \\
\text { bandanas, t-shirt and package of } \\
\text { resources. }\end{array}$ & $\begin{array}{l}\text { Administrated } \\
\text { questionnaires }\end{array}$ & $\begin{array}{l}\text { Survey1 } \\
\text { (Early 2005) } \\
\text { Survey 2: } \\
\text { After } \\
\text { BRIDGE } \\
\text { PROJECT } \\
\text { (December } \\
\text { 2005) }\end{array}$ & $\begin{array}{l}\text { Survey } 1(890,54.3 \% \text { Female }) \\
\text { Survey } 2(881,50 \% \text { Males }) \\
\text { Survey on different samples on } \\
\text { the same villages. }\end{array}$ & $\begin{array}{l}\text { Self-efficacy significantly } \\
\text { associated with the behaviour of } \\
\text { willingness to get HIV tested } \\
\text { ( } \mathrm{B}=0.49, p<0.001) \text {. }\end{array}$ \\
\hline $\begin{array}{l}\text { Burke LE, et. } \\
\text { al., } 2005 \\
\text { USA }\end{array}$ & $\begin{array}{l}\text { Adherence to a } \\
\text { cholesterol-lowering } \\
\text { diet }\end{array}$ & $\begin{array}{l}\text { Coronary heart } \\
\text { disease }\end{array}$ & $\begin{array}{l}\text { Experiment (Randomized } \\
\text { controlled trial with } 2 \text { arms) } \\
\text { Some intervention control group } \\
\text { Physician discussion, verbal } \\
\text { persuasion, counselling and health } \\
\text { information on individual tailored } \\
\text { telephone intervention }\end{array}$ & $\begin{array}{l}\text { Questionnaires } \\
\text { 38-question Connor } \\
\text { DHS (Dietary } \\
\text { adherence assessment) } \\
\text { Three-Day Food } \\
\text { record } \\
\text { Cholesterol-Lowering } \\
\text { Diet Self-efficacy } \\
\text { Scales (CLDSES) } \\
\text { Perceived Therapeutic } \\
\text { Efficacy Scale } \\
\text { Serum lipids profile }\end{array}$ & 14 weeks & $\begin{array}{l}65 \text { patients diagnosed with } \\
\text { hypercholesterolemia } \\
\text { Intervention }(65 \% \text { Male), } \\
\text { Usual care ( } 62 \% \text { Male). } \\
\text { Mean age } \\
\text { (Intervention=53.74), Usual } \\
\text { care=55.9) }\end{array}$ & $\begin{array}{l}\text { Self-efficacy was not a mediator } \\
\text { of improving adherence } \\
\text { behaviour to a } \\
\text { cholesterol-lowering diet. }\end{array}$ \\
\hline
\end{tabular}




\begin{tabular}{|c|c|c|c|c|c|c|c|}
\hline $\begin{array}{l}\text { Iever-Landis } \\
\text { CE, et. al, } \\
2003 \\
\text { USA }\end{array}$ & 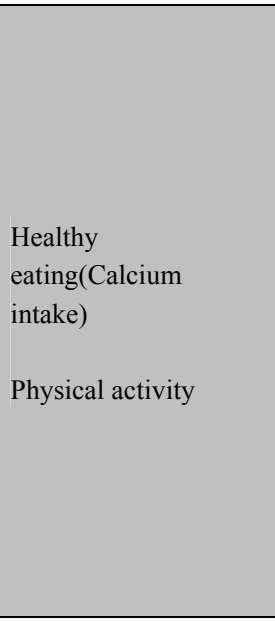 & Osteoporosis & $\begin{array}{l}\text { Observational (Cross sectional } \\
\text { intervention study) } \\
\text { One-off surveys based on previous } \\
\text { health promotion program } \\
\text { Educational training sessions } \\
\text { (Lectures, demonstrations and } \\
\text { group discussions) }\end{array}$ & $\begin{array}{l}\text { Administered food } \\
\text { frequency } \\
\text { questionnaires } \\
\text { Administered } \\
\text { questionnaires for } \\
\text { physical activity } \\
\text { Questionnaires for } \\
\text { osteoporosis } \\
\text { prevention knowledge } \\
\text { and self-efficacy diet } \\
\text { Children physical } \\
\text { activity questionnaires } \\
\text { (CPAQ) }\end{array}$ & 3 months & $\begin{array}{l}354 \text { preadolescents girls } \\
\text { Recruited for participation } \\
\text { from the Girl Scouts of Lake } \\
\text { Erie Council } \\
\text { Mean age }=9.37 \\
84.2 \% \text { Caucasian }\end{array}$ & $\begin{array}{l}\text { For family support, self-efficacy } \\
\text { significantly predicted dietary } \\
\text { intake of calcium ( } \mathrm{B}=0.25 \text {, } \\
p<0.001 \text { ). Self-efficacy was not } \\
\text { significantly predicted frequency } \\
\text { of exercise activity. } \\
\text { For friend support, self-efficacy } \\
\text { was not significantly predicted } \\
\text { calcium intake behaviour. } \\
\text { Self-efficacy for Ca intake does } \\
\text { function as a partial mediator of } \\
\text { the relationship between family } \\
\text { support for exercise and Ca } \\
\text { intake behaviour. } \\
\text { Self-efficacy was not a predictor } \\
\text { to exercise behaviour. }\end{array}$ \\
\hline $\begin{array}{l}\text { Burke V., et. } \\
\text { al., } 2008 \\
\text { Australia }\end{array}$ & $\begin{array}{l}\text { Healthy eating } \\
\text { (reduction in saturated } \\
\text { fat consumption) } \\
\text { Exercise }\end{array}$ & Hypertension & $\begin{array}{l}\text { Experiment (Randomised } \\
\text { controlled trial with } 2 \text { arms) } \\
\text { Some intervention control group } \\
\text { Individual discussion, newsletters, } \\
\text { physical activity group session, } \\
\text { publications and seminars. }\end{array}$ & $\begin{array}{l}\text { Questionnaires } \\
\text { Diet records } \\
\text { Revised Ways of } \\
\text { Coping Checklist }\end{array}$ & $\begin{array}{l}4 \text { months } \\
\text { (post } \\
\text { intervention) } \\
1 \text { year } \\
\text { (follow up) }\end{array}$ & $\begin{array}{l}241 \text { (118 usual care, } 123 \\
\text { program group) recruited by } \\
\text { advertisement. } \\
100 \% \text { Caucasian } \\
55 \% \text { Female } \\
\text { Age } 40 \text { to } 70 \text { years old } \\
\text { BMI }>26 \mathrm{~kg} / \mathrm{m} \\
\text { Treated with one or two } \\
\text { antihypertensive drugs for at } \\
\text { least } 3 \text { months }\end{array}$ & $\begin{array}{l}\text { Self-efficacy was significantly } \\
\text { associated with reduction in } \\
\text { saturated fat consumption at post } \\
\text { intervention (Effect size }-0.055 \text {, } \\
95 \% \text { CI: }-0.125,-0.005 \text { ). } \\
\text { Self-efficacy explained change in } \\
\text { time spent in exercise of at least } \\
\text { moderate intensity at post } \\
\text { intervention (Effect size } 0.059 \text {, } \\
95 \%, \text { CI: } 0.003,0.147) \text {. }\end{array}$ \\
\hline
\end{tabular}




\begin{tabular}{|c|c|c|c|c|c|c|c|}
\hline $\begin{array}{l}\text { Strathdee } \\
\text { SA., et. al, } \\
2009 \\
\text { Mexico }\end{array}$ & Use of condom & HIV/ AIDS & $\begin{array}{l}\text { Observational (Cross-sectional } \\
\text { study) } \\
\text { One-off surveys based on previous } \\
\text { health promotion program } \\
\text { Individual counselling session } \\
\text { integrating motivational } \\
\text { interviewing and principles of } \\
\text { behavioural change }\end{array}$ & $\begin{array}{l}\text { Interviewer } \\
\text { administrated } \\
\text { questionnaires }\end{array}$ & $\begin{array}{l}2 \text { years for } \\
\text { data } \\
\text { collection } \\
6 \text { months } \\
\text { follow up } \\
\text { visit }\end{array}$ & $\begin{array}{l}409 \text { HIV- female sex workers } \\
16 \% \text { had injected drugs } \\
84 \% \text { returned for a 6-month } \\
\text { follow up visits } \\
\text { Age at least } 18 \text { years old } \\
\text { (Median=32) and having } \\
\text { traded sex for drugs, money or } \\
\text { other material benefits within } \\
\text { the previous } 2 \text { months }\end{array}$ & $\begin{array}{l}\text { Greater increase self-efficacy } \\
\text { was associated with greater } \\
\text { increase in percentage of condom } \\
\text { use ( } t=2.66, p=0.008) \text {. } \\
\text { Self-efficacy was associated with } \\
\text { change in condom use regardless } \\
\text { of injection status (injection drug } \\
\text { use was not a mediator between } \\
\text { self-efficacy and condom use). }\end{array}$ \\
\hline $\begin{array}{l}\text { Critchley CR, } \\
\text { et. al, } 2012 \\
\text { Australia }\end{array}$ & $\begin{array}{l}\text { Healthy eating } \\
\text { Physical activity }\end{array}$ & $\begin{array}{l}\text { Type-2 Diabetes } \\
\text { Mellitus }\end{array}$ & $\begin{array}{l}\text { Experiment (Randomised } \\
\text { Controlled Trial with } 2 \text { arms) } \\
\text { Some intervention control group } \\
\text { Group-based intervention (small } \\
\text { group sessions, } \\
\text { psychosocial-educational, } \\
\text { information-giving sessions on } \\
\text { diabetes, diet, exercise and } \\
\text { behavioural change). }\end{array}$ & $\begin{array}{l}\text { GP's records } \\
\text { Questionnaires } \\
\text { International Physical } \\
\text { Activity Scale } \\
\text { Food Choices } \\
\text { Questionnaires }\end{array}$ & 6 months & $\begin{array}{l}307 \text { adults } \\
59 \% \text { Female } \\
\text { Mean age }=62.5(\mathrm{SD}=10.1) \\
208 \text { (Intervention) and } 99 \\
\text { (control) } \\
\text { Diagnosed with pre-diabetes }\end{array}$ & $\begin{array}{l}\text { Self-efficacy was not a mediator } \\
\text { between participation in program } \\
\text { and change in physical activity } \\
\text { and diet behaviour. }\end{array}$ \\
\hline $\begin{array}{l}\text { Fuchs R., et. } \\
\text { al., } 2012 \\
\text { Germany }\end{array}$ & Exercise behaviour & $\begin{array}{l}\text { Chronic } \\
\text { orthopaedic } \\
\text { conditions } \\
\text { (arthritis, } \\
\text { chronic back } \\
\text { pain, etc) }\end{array}$ & $\begin{array}{l}\text { Experiment (Randomised } \\
\text { controlled trial with } 2 \text { arms) } \\
\text { No treatment control group } \\
\text { MoVo-LISA Program (Group } \\
\text { meetings, one-to-one interviews, } \\
\text { postal reminder, telephone call) }\end{array}$ & Questionnaires & 12 months & $\begin{array}{l}1113 \text { patients of chronic } \\
\text { orthopaedic conditions } \\
\text { recruited from in-patient } \\
\text { rehabilitation program. } \\
681 \text { (control) and } 432 \\
\text { (intervention) } \\
\text { Mean age }=51.1(\mathrm{SD}=6.9)\end{array}$ & $\begin{array}{l}\text { Self-efficacy was not directly } \\
\text { predicted exercise behaviour, but } \\
\text { indirectly with mediation of } \\
\text { intention strength and } \\
\text { self-concordance. }\end{array}$ \\
\hline
\end{tabular}




\begin{tabular}{|c|c|c|c|c|c|c|c|}
\hline $\begin{array}{l}\text { Sharma SV., } \\
\text { et. al., } 2010 \\
\text { USA }\end{array}$ & $\begin{array}{l}\text { Healthy eating } \\
\text { (Calcium intake } \\
\text { behaviour) }\end{array}$ & Osteoporosis & $\begin{array}{l}\text { Descriptive study based on } \\
\text { secondary data of the previous } \\
\text { intervention study } \\
\text { One-off surveys based on previous } \\
\text { health promotion program } \\
\text { Based on The Incorporating More } \\
\text { Physical Activity and Calcium in } \\
\text { Teens (IMPACT) Program } \\
\text { (quasi-experimental, school-based } \\
\text { nutrition and physical activity } \\
\text { intervention). }\end{array}$ & $\begin{array}{l}\text { Anthropometric } \\
\text { measures } \\
\text { Quantitative } \\
\text { ultrasound for bone } \\
\text { quality } \\
\text { Food frequency } \\
\text { questionnaires } \\
\text { Calcium, osteoporosis } \\
\text { and physical activity } \\
\text { (COPA) } \\
\text { questionnaires }\end{array}$ & $\begin{array}{l}\text { Based on } \\
\text { previous } \\
\text { intervention } \\
\text { ( } 2 \text { years) }\end{array}$ & $\begin{array}{l}717 \text { girls from } 12 \text { schools } \\
\text { (sixth grade girls) } \\
\text { Mean age }=11.6(\mathrm{SD}=0.4) \\
72 \% \text { were non-Hispanic white }\end{array}$ & $\begin{array}{l}\text { Self-efficacy was not } \\
\text { significantly predicted calcium } \\
\text { intake behaviour } \\
\text { Self-efficacy has indirect } \\
\text { relationship with calcium intake } \\
\text { behaviour with mediation of } \\
\text { outcome expectations }(\mathrm{B}=0.85 \text {, } \\
p<0.05) \text {. }\end{array}$ \\
\hline $\begin{array}{l}\text { Chariyeva Z., } \\
\text { et. al, } 2011 \\
\text { USA }\end{array}$ & Safer sex practice & HIV/ AIDS & $\begin{array}{l}\text { Experiment (Randomised } \\
\text { controlled trial with } 2 \text { arms). } \\
\text { Historical control group } \\
\text { Safe Talk program (session heart } \\
\text { healthy nutrition counselling } \\
\text { program, individual counselling, } \\
\text { CD, workbook series) }\end{array}$ & $\begin{array}{l}\text { Computer-assisted } \\
\text { self-interview } \\
\text { (ACASI) in the clinics }\end{array}$ & $\begin{array}{l}\text { Based on } \\
\text { previous } \\
\text { program (12 } \\
\text { months) }\end{array}$ & $\begin{array}{l}183 \mathrm{HIV} \text { infected patients } \\
\text { receiving care at clinics } \\
\text { Mean age }=41.9(9.58) \\
73.3 \% \text { African American } \\
62.3 \% \text { Male }\end{array}$ & $\begin{array}{l}\text { Self-efficacy was negatively } \\
\text { associated with sexual practices } \\
\text { (Unprotected sexual intercourse } \\
\text { behaviour decreased as } \\
\text { self-efficacy to practice safer sex } \\
\text { increased). } \\
\text { A one unit increase in } \\
\text { self-efficacy was associated with } \\
\text { a } 52 \text { and } 39 \% \text { decreased in } \\
\text { unprotected sexual intercourse } \\
\text { activity. } \\
\text { Self-efficacy was a mediator } \\
\text { between number of counselling } \\
\text { sessions and dose of unprotected } \\
\text { sexual intercourse. }\end{array}$ \\
\hline
\end{tabular}


Apart from variation in the type of disease, settings, participants and educational component, the included studies also presented different types of health behaviours to change, which were promoted during the intervention. Most of studies were experimental in nature (9 papers) and 3 studies were observational with one study being primarily descriptive. The exact mix of interventions varied between studies. Four different approaches were used in the study designs: 1 study used an historical control period, measuring outcomes in the same setting at baseline and at a later time point, 2 studies included a control arm with no additional treatment as well as an intervention arm, 6 studies included a control arm exposed to some health promotion activity, along with an intervention arm and 4 studies are categorized as one-off survey following health promotion and educational intervention. The topics covered by the studies included dengue survey practices (one study), medication taking behaviour (one study), breast self-examination behavior (one study), safe sex and HIV test behaviour (one study), health eating with physical activity behavior (one study), eating behaviour only (one study) and physical activity(one study).

Most papers (8 studies) are mainly on non-communicable diseases (breast cancer, coronary heart disease, osteoporosis, hypertension, and type- 2 diabetes. The remaining 3 papers have a range of communicable diseases such as dengue fever, tuberculosis and HIV/AIDS. Studies varied with regard to types of health promotion and education intervention. Most of studies applied a group of educational activities including use of print or electronic media, group discussions or training sessions, counseling or meeting sessions, demonstrations or talks, individual advice and behavioural contract.

All studies used questionnaires (4 administrated and 9 self-administrated) and other behaviour-related tool to measure the outcome. To measure self-efficacy, all studies used 5-points Likert Scale format questionnaires which were either pre validated or adapted from previous studies. However, there are a variety of tools used to measure health related behaviour; larval survey form, diet recall form or food record, physician prescription of lifestyle change, Block 2005 Food Frequency, pedometer, children physical activity questionnaires (CPAQ), calcium, osteoporosis and physical activity questionnaires (COPA), physical activity recall (3DPAR) and computer-assisted self-interview (ACASI). Most of these health behaviour tools of measurement were in self-reporting format.

\subsection{Study Validity and Limitations}

The potential limitations and factors that may influence validity of each individual study are described in Table 2. Among the issues affecting the overall dataset are the studies that relied on self-reported, and therefore prone to reporting bias, measures on eating and physical activity behavior (Chariyeva et al., 2013; Critchley, Hardie, \& Moore, 2012; Luszczynska, 2004; Piaseu, Schepp, \& Belza, 2002; Strathdee et al., 2009). Selective sampling in recruitment to a particular group of participants (Ievers-Landis et al., 2003; Piaseu et al., 2002; Sharma et al., 2010) or being geographically limited thus reducing generalizability of the study.

Attrition bias resulted from participants being lost to follow-up or the ability to complete the measurement of self-efficacy and/or health behaviour change. The response rate varied from $61 \%$ to $97 \%$, but there are 2 studies that did not clearly highlight the issue (Sharma et al., 2010; Therawiwat, Fungladda, Kaewkungwal, Imamee, \& Steckler, 2005). Multivariate analysis was the main analysis method performed by most studies. Four studies did not have any statistical adjustment because either they compared the intervention and control group using t-test (L. E. Burke, Dunbar-Jacob, Orchard, \& Sereika, 2005) or they analysed the data for mediating and moderating effects or path analysis (Fuchs, Seelig, Göhner, Burton, \& Brown, 2012; Luszczynska, 2004; Sharma et al., 2010). Demographics like age, ethnic, sex and education level are among the most factors included for statistical adjustment.

Another important issue here is selective outcome reporting. Not all the results may have been made available in the published version even though the studies measured many outcomes in wide range. Many studies were reported in different ways as categorical or continuous outcome which made it incomparable to assess the findings.

\subsection{Self-Efficacy and Communicable Disease Control}

One study investigated the effect of self-efficacy on dengue survey practices following community-based health promotion interventions in Thailand (Therawiwat et al., 2005). The programme was associated with a reduction in entomological indices of mosquito breeding and also with increased knowledge, perception and self-efficacy. However, in a multiple predictor model self-efficacy did not predict larval survey practices ( $p>0.05)$.

Three studies evaluated the impact of self-efficacy on behaviours related to control or prevention of HIV/AIDS transmission, two cross-sectional studies (Berendes \& Rimal, 2011; Strathdee et al., 2009) and one two-armed 
randomized controlled trial study (Chariyeva et al., 2013). The first study evaluated self-efficacy as a facilitator of uptake of HIV testing behaviour following a communication-based behaviour change programme in Malawi (Berendes \& Rimal, 2011). In both adjusted and unadjusted analysis, self-efficacy which was measured based on the individual's efficacy to remain abstinent (if not in a relationship), to remain faithful to one's partner, and to discuss and insist on use of condom was significantly associated with the previous testing behaviour or if a person had not yet been tested, with the desire to be tested. Self-efficacy was significantly associated with the willingness to get HIV tested $(\mathrm{p}<0.001)$. The second is study determined whether self-efficacy and injecting drug user status predicted condom use behaviour among Mexican female sex workers (Strathdee et al., 2009). A greater increase in self-efficacy was associated with greater condom use $(\mathrm{p}<0.05)$ regardless of injection drug use status. The third study assessed the mediation effect of self-efficacy and motivation on safer sex play practices among 490 People Living with HIV/AIDS (PLWHA) (Chariyeva et al., 2013). Self-efficacy was negatively associated with risky sexual practices. A one unit increased in self-efficacy was associated with $39 \%$ decreased in unprotected sexual intercourse activity. Moreover, self-efficacy was a mediator between number of counseling sessions and degree of unprotected sexual intercourse.

In a study of tuberculosis, self-efficacy was associated with medication-taking behaviour to control transmission (Morisky et al., 2001). This randomized study showed that self-efficacy strongly correlated with the completion of care $(p<0.001)$. Peer counseling had a significant impact on self-efficacy for medication taking and on medication-taking-behaviour.

\subsection{Self-Efficacy and Non-Communicable Disease Control}

Seven studies examined the relationship between self-efficacy and behaviour related to healthy eating and/or exercise or physical activities to non-communicable control diseases; three studies were on osteoporosis prevention (Ievers-Landis et al., 2003; Piaseu et al., 2002; Sharma et al., 2010) and the rest are on coronary heart disease (L. E. Burke et al., 2005) hypertension, type-2 diabetes (Critchley et al., 2012) ${ }^{3}$ and chronic orthopaedic conditions like arthritis (Fuchs et al., 2012). There is also one study related to breast self-examination behavior (Luszczynska, 2004).

The first study assessed self-efficacy using a osteoporosis self-efficacy scale (OSES) to measure confidence in women aged 21 years or younger to perform osteoporosis risk reduction behaviours focusing on exercise and dietary calcium intake (Schwarzer, Luszczynska, Ziegelmann, Scholz, \& Lippke, 2008). After intervention, self-efficacy was found to directly predict calcium intake behaviour $(p<0.05)$ and exercise $(p<0.01)$. However, calcium intake was indirectly predicted by knowledge through health belief attitudes and self-efficacy. Path coefficient and $R^{2}$ statistics of causal analysis showed that self-efficacy was a mediator between attitudes and calcium intake behaviour $(\mathrm{p}<0.05)$, which accounted for $30 \%$ of variance as well as between knowledge and exercise $(\mathrm{p}<0.001)$, which accounted for $45 \%$ of the variance.

The second study on osteoporosis was a cross-sectional study among middle school girls to identify pathways by which psychosocial factors influence calcium intake and bone quality (Sharma et al., 2010). Confirmatory factor analysis and path analysis showed that self-efficacy did not predict calcium intake $(\mathrm{p}>0.05)$. However, self-efficacy had a significant indirect effect in calcium intake behaviour with mediation of outcome expectations $(\mathrm{p}<0.05)$. This indirect influence could be because participants with a higher confidence toward choosing calcium-containing foods could have higher outcome expectations, which could lead to higher calcium intake practices.

The third study regarding prevention of osteoporosis was carried out among 354 girls of a scout troop ages between 8-11 years old (Ievers-Landis et al., 2003). This study tried to predict the influence of self-efficacy and exercise self-efficacy based on Bandura's social cognitive theory. Even though self-efficacy significantly predicted dietary intake of calcium in presence of family support $(\mathrm{p}<0.001)$, it was not a significant predictor of frequency of exercise activity ( $>0.05)$. However, a structural equation model found that, self-efficacy for calcium intake functioned as a partial mediator in the relationship between family support for exercise and calcium intake behaviour.

A randomized controlled trial investigated self-efficacy using cholesterol lowering diet self-efficacy scale (CLDSES) and adherence to a cholesterol lowering diet behavior (L. E. Burke et al., 2005). Participants were patients with hypercholesterolemia selected from three academic medical centre practice and research sites. Participants were given low cholesterol diet and/or pharmacological therapy for at least 2 weeks. Adherence behaviour was examined by comparing actual intake of total and saturated fat and cholesterol to prescribed amounts. Result showed that baseline self-efficacy significantly associated with concurrent adherence scores within the intervention group for total fat $(p<0.05)$, saturated fat $(p<0.01)$ and cholesterol $(p<0.05)$. However, the 
control group only intake of cholesterol diet significantly associated with baseline self-efficacy $(\mathrm{p}<0.05)$. After intervention, the only adherence scores that were inversely related to self-efficacy were saturated fat for the intervention group, and saturated fat and cholesterol for the usual care group. The study assumed that self-efficacy was not a mediator of improving adherence behaviour to a low cholesterol diet in controlling coronary heart disease.

Another randomized controlled trial of a cognitive lifestyle program for drug-treated overweight hypertensive patients in Perth, Australia reported that change in self-efficacy mediated change in saturated fat consumption (effect size (ES) $-0.055,95 \% \mathrm{CI}(-0.125,-.005)$ at post intervention and (ES $-0.054,95 \% \mathrm{CI} ; 0.127,-0.005)$ at the follow up (V. Burke, Beilin, Cutt, Mansour, \& Mori, 2007). Self-efficacy also explained change in time spent in exercise at least moderate intensity at post intervention (ES 0.059, 95\% CI; 0.003, 0.147).

Critchley et al. conducted a randomized control trial examining the psychological process of lifestyle change among 307 pre-diabetes adults following a six-session group-based intervention to promote healthier living (Critchley et al., 2012). They reported that, despite the fact that participation in the six small group's psychosocial-educational sessions was significantly associated with improvement in self-efficacy, it did not become a mediator between this participation and change in healthy diet and physical activity behaviour. Result also showed that knowledge about diabetes $(\mathrm{p}<0.05)$ significantly contributed to the effect of program participation on increased physical activity. However, none of those psychosocial variables significantly mediated change on eating behaviour.

A quasi-experiment longitudinal study on the cognitive mediation effects of a group-based intervention on exercise behaviour in 220 adults with chronic orthopedic conditions concluded that, self-efficacy did not directly predict exercise behavior (Fuchs et al., 2012). However, self-efficacy mediated the impact of intention strength and self-concordance.

Luszcynska et al. reported a cluster randomised trial for promotion of breast self-examination to reduce mortality from breast cancer amongst 417 female students in Poland (Luszczynska, 2004). This study concluded that maintenance self-efficacy predicted change in breast self-examination performance $(\mathrm{p}<0.05)$ as well as intention and planning in the intervention group. Pre-action self-efficacy however, only significant in predicting intention to do breast self-examination in both intervention and control groups.

\section{Discussions}

Previous reviews have generally focused only on the impact of health promotion on self-efficacy but not whether that self-efficacy has a subsequent impact on behavioural change (Brown \& Summerbell, 2009; Olander et al., 2013; Yehle \& Plake, 2010) or on general healthy living rather than specific disease risk reduction (Lubans, Foster, \& Biddle, 2008; Plotnikoff, Costigan, Karunamuni, \& Lubans, 2013; Rhodes \& Dickau, 2012; Strecher, McEvoy DeVellis, Becker, \& Rosenstock, 1986).

From the total 13 included studies, eight were found a direct association between self-efficacy and specific health behaviours (Berendes \& Rimal, 2011; V. Burke et al., 2007; Chariyeva et al., 2013; Ievers-Landis et al., 2003; Luszczynska, 2004; Morisky et al., 2001; Piaseu et al., 2002; Strathdee et al., 2009). Seven studies analysed for mediation effects of self-efficacy on health behaviour (V. Burke et al., 2007; Chariyeva et al., 2013; Critchley et al., 2012; Ievers-Landis et al., 2003; Luszczynska, 2004; Piaseu et al., 2002; Sharma et al., 2010) and of these six showed significant mediation effect between other psychological variables and health behaviour.

Although the studies reviewed generally conceptualized self-efficacy as perceived capabilities of performing specific health behaviours, operational measures of self-efficacy varied considerably. A two-step approach was used to measure self-efficacy by determining a person belief on behaviour modification could be accomplished and to rate the strength of the belief (Albert Bandura, 1997). In many of the studies, this method was incorporated into a unified set of measures that included both strength and level of self-efficacy. In most studies, participants were asked how confident they would feel in performing targeted health behaviour in different situations where the ability to perform the behaviour might vary accordingly.

In studies where other psychosocial constructs like family support, attitudes, intention strength and self-concordance, as well as outcome expectations were examined, self-efficacy was found to be a distinct and powerful predictor for health behavior (Fuchs et al., 2012; Ievers-Landis et al., 2003; Piaseu et al., 2002; Sharma et al., 2010). Other findings suggest that it interact with or influence self-efficacy to change or maintain targeted health behaviour to control diseases. Self-efficacy may depend on perceived influence in controlling outcomes. This supports Bandura's Social Learning Theory that psychosocial arousal influences self-efficacy and self-efficacy reflect a person's perceived abilities rather than one's true abilities that often influence behaviour 
change and maintenance (A Bandura, 1977).

Perceptions of susceptibility to a particular illness and of benefits of taking recommended preventive actions could both be considered expectations of outcome. Outcome expectations are a major component of value-expectancy models of health behaviour such as the Health Belief Model (Becker, 1974; Janz \& Becker, 1984). These expectations may play the largest role in influencing initial motivation and decision to change a health practice (Sharma et al., 2010). Health practices which are not particularly difficult to modify but whose perceive consequences are uncertain may largely depend on outcome expectations like compliance to treatment in tuberculosis (Morisky et al., 2001). Studies have found that outcome expectations, such as perceived susceptibility to tuberculosis-related illness or perceived benefits of taking the medication, are associated with compliance to tuberculosis treatment (Morisky et al., 2001).

In situation where belief and trust play a major role among sexual worker, self-efficacy is inadequate to mediate the safe health practice in using condoms to prevent sexually transmitted diseases (Chariyeva et al., 2013; Strathdee et al., 2009). Finally, both outcome and self-efficacy may be required to explain behaviour change process when there is a need for a change in practices of uncertain consequences. This might be applied during the modification of certain dietary habits, such as reducing the intake of saturated fat to reduce risk of hypertensive complications like stroke (V. Burke et al., 2007) or reducing foods that high in cholesterol to reduce risk of coronary heart disease (L. E. Burke et al., 2005).

The findings of this review support the perspective of Bandura's Social Learning Theory regarding the concept that self-efficacy relates to beliefs about abilities to change specific behaviours in particular situations (Schunk \& Carbonari, 1984). Self-efficacy does not refer to a personality characteristic or a global trait that operates independently of contextual factors (A Bandura, 1977; Albert Bandura, 1978). The individual's self-efficacy will vary greatly depending on the particular task and context. Therefore, it is inappropriate to characterize an individual as having 'high' or 'low' self-efficacy without reference to the specific health behaviour and circumstance with which self-efficacy judgment is associated, i.e. control or prevent any particular diseases. The finding of this review also supports the previous studies on the predictive value of self-efficacy has on health behaviour change either direct effects or as an effective mediator between other psychological variables with health behaviours (Baldwin et al., 2006; Darker et al., 2010; Hendriksen et al., 2007; Motl et al., 2002; Oei \& Burrow, 2000; Scholz et al., 2005; Strauss et al., 2001).

\section{Conclusions}

In conclusion, the majority of the studies identified in this review found a significant association between self-efficacy in both short and long term success of health promotion interventions. The investigators had undertaken the appropriate analyses to show the effect of self-efficacy on psychological factors and behavioural change. This review supports the hypothesis that the concept of self-efficacy is a good psychosocial construct that can translate knowledge and information from health promotion and educational intervention into behavioural action. Improving self-efficacy must be one of main agenda in successful behavioural-impact health promotion and educational interventions to control and prevent diseases. Future health promotion and educational intervention to control or prevent diseases must be designed to influence related health behaviour by giving more attention on enhancing self-efficacy implicitly.

\section{Ethics Approval and Consent to Participate}

Since only official public data were used in this study, neither institutional board approval nor informed consent was required.

\section{Acknowledgements}

We would like to thank the Director General of Health, Malaysia for the permission to publish this paper.

\section{Authors' Contribution}

AI conceptualized and coordinated the study and drafted the manuscript. NAM and NM aided in the study design and ensured that all the elements of the protocol were reflected in the manuscript. MHAM and NAL contributed to the design and reviewed the contributions. MFB, MSMR and ZZ reviewed the content of the protocol and SSM reviewed and edited the manuscript. All authors read and approved the final version.

\section{Competing Interests Statement}

All authors declare no conflict of interest. 


\section{References}

Ashford, S., Edmunds, J., \& French, D. P. (2010). What is the best way to change self - efficacy to promote lifestyle and recreational physical activity? A systematic review with meta - analysis. British journal of health psychology, 15(2), 265-288. https://doi.org/10.1348/135910709X461752

Baldwin, A. S., Rothman, A. J., Hertel, A. W., Linde, J. A., Jeffery, R. W., Finch, E. A., \& Lando, H. A. (2006). Specifying the determinants of the initiation and maintenance of behavior change: an examination of self-efficacy, satisfaction, and smoking cessation. Health Psychology, 25(5), 626. https://doi.org/10.1037/0278-6133.25.5.626

Bandura, A. (1977). Social Learning Theory: Prentice Hall. Englewood Cliffs, NJ.

Bandura, A. (1978). Self-efficacy: Toward a unifying theory of behavioral change. Advances in Behaviour Research and Therapy, 1(4), 139-161. https://doi.org/10.1016/0146-6402(78)90002-4

Bandura, A. (1997). Self-efficacy: The exercise of control. Macmillan.

Bandura, A., \& Wood, R. (1989). Effect of perceived controllability and performance standards on self-regulation of complex decision making. Journal of personality and social psychology, 56(5), 805. https://doi.org/10.1037/0022-3514.56.5.805

Baranowski, T., Anderson, C., \& Carmack, C. (1998). Mediating variable framework in physical activity interventions: How are we doing? How might we do better? American journal of preventive medicine, 15(4), 266-297. https://doi.org/10.1016/S0749-3797(98)00080-4

Becker, M. H. (1974). The health belief model and personal health behavior. Health education monographs, 2(4), 409-419. https://doi.org/10.1177/109019817400200407

Berendes, S., \& Rimal, R. N. (2011). Addressing the slow uptake of HIV testing in Malawi: the role of stigma, self-efficacy, and knowledge in the Malawi BRIDGE Project. Journal of the Association of Nurses in AIDS Care, 22(3), 215-228. https://doi.org/10.1016/j.jana.2010.08.005

Bouffard-Bouchard, T. (1990). Influence of self-efficacy on performance in a cognitive task. The Journal of Social Psychology, 130(3), 353-363. http://dx.doi.org/10.1080/00224545.1990.9924591

Brega, A. G., Pratte, K. A., Jiang, L., Mitchell, C. M., Stotz, S. A., Loudhawk-Hedgepeth, C., . . Beals, J. (2013). Impact of targeted health promotion on cardiovascular knowledge among American Indians and Alaska Natives. Health Educ Res, 28(3), 437-449. https://doi.org/10.1093/her/cyt054

Brown, T., \& Summerbell, C. (2009). Systematic review of school - based interventions that focus on changing dietary intake and physical activity levels to prevent childhood obesity: an update to the obesity guidance produced by the National Institute for Health and Clinical Excellence. Obesity reviews, 10(1), 110-141. https://doi.org/10.1111/j.1467-789X.2008.00515.x

Burke, L. E., Dunbar-Jacob, J., Orchard, T. J., \& Sereika, S. M. (2005). Improving adherence to a cholesterol-lowering diet: a behavioral intervention study. Patient education and counseling, 57(1), $134-142$. https://doi.org/10.1016/j.pec.2004.05.007

Burke, V., Beilin, L. J., Cutt, H. E., Mansour, J., \& Mori, T. A. (2007). Moderators and mediators of behaviour change in a lifestyle program for treated hypertensives: a randomized controlled trial (ADAPT). Health Educ Res, 23(4), 583-591. https://doi.org/10.1093/her/cym047

Chaffee, S. H., \& Roser, C. (1986). Involvement and the consistency of knowledge, attitudes, and behaviors. Communication research, 13(3), 373-399. https://doi.org/10.1177/009365086013003006

Chariyeva, Z., Golin, C. E., Earp, J. A., Maman, S., Suchindran, C., \& Zimmer, C. (2013). The role of self-efficacy and motivation to explain the effect of motivational interviewing time on changes in risky sexual behavior among people living with HIV: a mediation analysis. AIDS and Behavior, 17(2), 813-823. https://doi.org/10.1007/s10461-011-0115-8

Critchley, C. R., Hardie, E. A., \& Moore, S. M. (2012). Examining the psychological pathways to behavior change in a group-based lifestyle program to prevent type 2 diabetes. Diabetes Care, 35(4), 699-705. https://doi.org/10.2337/dc11-1183

Darker, C., French, D., Eves, F., \& Sniehotta, F. (2010). An intervention to promote walking amongst the general population based on an 'extended'theory of planned behaviour: a waiting list randomised controlled trial. Psychology and Health, 25(1), 71-88. http://dx.doi.org/10.1080/08870440902893716 
Ezedinachi, E., Ross, M., Meremiku, M., Essien, E., Edem, C., Ekure, E., \& Ita, O. (2002). The impact of an intervention to change health workers' HIV/AIDS attitudes and knowledge in Nigeria: a controlled trial. Public health, 116(2), 106-112. https://doi.org/10.1038/sj.ph.1900834

Fuchs, R., Seelig, H., Göhner, W., Burton, N. W., \& Brown, W. J. (2012). Cognitive mediation of intervention effects on physical exercise: Causal models for the adoption and maintenance stage. Psychology \& health, 27(12), 1480-1499. http://dx.doi.org/10.1080/08870446.2012.695020

Hendriksen, E. S., Pettifor, A., Lee, S.-J., Coates, T. J., \& Rees, H. V. (2007). Predictors of condom use among young adults in South Africa: The reproductive health and HIV research unit national youth survey. American Journal of Public Health, 97(7), 1241-1248. https://doi.org/10.2105/AJPH.2006.086009

Hyde, J., Hankins, M., Deale, A., \& Marteau, T. M. (2008). Interventions to increase self-efficacy in the context of addiction behaviours: a systematic literature review. Journal of Health Psychology, 13(5), 607-623. https://doi.org/10.1177/1359105308090933

Ievers-Landis, C. E., Burant, C., Drotar, D., Morgan, L., Trapl, E. S., \& Kwoh, C. K. (2003). Social support, knowledge, and self-efficacy as correlates of osteoporosis preventive behaviors among preadolescent females. Journal of Pediatric Psychology, 28(5), 335-345. https://doi.org/10.1093/jpepsy/jsg023

Janz, N. K., \& Becker, M. H. (1984). The health belief model: A decade later. Health education quarterly, 11(1), 1-47. https://doi.org/10.1177/109019818401100101

Lewis, B. A., Marcus, B. H., Pate, R. R., \& Dunn, A. L. (2002). Psychosocial mediators of physical activity behavior among adults and children. American journal of preventive medicine, 23(2), 26-35. https://doi.org/10.1016/S0749-3797(02)00471-3

Lubans, D. R., Foster, C., \& Biddle, S. J. (2008). A review of mediators of behavior in interventions to promote physical activity among children and adolescents. Preventive medicine, 47(5), 463-470. https://doi.org/10.1016/j.ypmed.2008.07.011

Luszczynska, A. (2004). Change in breast self-examination behavior: Effects of intervention on enhancing self-efficacy. International Journal of Behavioral Medicine, 11(2), 95-103. https://doi.org/10.1207/s15327558ijbm1102_5

Maibach, E., Flora, J. A., \& Nass, C. (1991). Changes in self-efficacy and health behavior in response to a minimal contact community health campaign. Health Communication, 3(1), 1-15. http://dx.doi.org/10.1207/s15327027hc0301_1

Marcus, B. H., King, T. K., Clark, M. M., Pinto, B. M., \& Bock, B. C. (1996). Theories and techniques for promoting physical activity behaviours. Sports medicine, 22(5), 321-331. https://doi.org/10.2165/00007256-199622050-00005

Mize, S. J., Robinson, B., Bockting, W., \& Scheltema, K. (2002). Meta-analysis of the effectiveness of HIV prevention interventions for women. AIDS care, 14(2), 163-180. http://dx.doi.org/10.1080/09540120220104686

Morisky, D. E., Malotte, C. K., Ebin, V., Davidson, P., Cabrera, D., Trout, P. T., \& Coly, A. (2001). Behavioral interventions for the control of tuberculosis among adolescents. Public Health Reports, 116(6), 568-574. https://doi.org/10.1016/S0033-3549(04)50089-4

Motl, R. W., Dishman, R. K., Saunders, R. P., Dowda, M., Felton, G., Ward, D. S., \& Pate, R. R. (2002). Examining social-cognitive determinants of intention and physical activity among black and white adolescent girls using structural equation modeling. Health Psychology, 21(5), 459. http://dx.doi.org/10.1037/0278-6133.21.5.459

Oei, T. P., \& Burrow, T. (2000). Alcohol expectancy and drinking refusal self-efficacy: a test of specificity theory. Addictive Behaviors, 25(4), 499-507. https://doi.org/10.1016/S0306-4603(99)00044-1

Olander, E. K., Fletcher, H., Williams, S., Atkinson, L., Turner, A., \& French, D. P. (2013). What are the most effective techniques in changing obese individuals' physical activity self-efficacy and behaviour: a systematic review and meta-analysis. International Journal of Behavioral Nutrition and Physical Activity, 10(1), 29. https://doi.org/10.1186/1479-5868-10-29

Pai, H.-H., Hong, Y.-J., \& Hsu, E.-L. (2006). Impact of a short-term community-based cleanliness campaign on the sources of dengue vectors: an entomological and human behavior study. Journal of Environmental Health, $68(6), 35-40$. 
Piaseu, N., Schepp, K., \& Belza, B. (2002). Causal analysis of exercise and calcium intake behaviors for osteoporosis prevention among young women in Thailand. Health Care for Women International, 23(4), 364-376. http://dx.doi.org/10.1080/0739933029008937

Plotnikoff, R. C., Costigan, S. A., Karunamuni, N., \& Lubans, D. R. (2013). Social cognitive theories used to explain physical activity behavior in adolescents: a systematic review and meta-analysis. Preventive medicine, 56(5), 245-253. https://doi.org/10.1016/j.ypmed.2013.01.013

Prochaska, J. O., \& DiClemente, C. C. (1982). Transtheoretical therapy: Toward a more integrative model of change. Psychotherapy: theory, research \& practice, 19(3), 276. http://dx.doi.org/10.1037/h0088437

Rhodes, R. E., \& Dickau, L. (2012). Moderators of the intention-behaviour relationship in the physical activity domain: a systematic review. Br J Sports Med, bjsports-2011-090411. http://dx.doi.org/10.1136/bjsports-2011-090411

Rogers, R. W. (1975). A protection motivation theory of fear appeals and attitude change1. The journal of psychology, 91(1), 93-114. doi:http://dx.doi.org/10.1080/00223980.1975.9915803

Scholz, U., Sniehotta, F. F., \& Schwarzer, R. (2005). Predicting physical exercise in cardiac rehabilitation: The role of phase-specific self-efficacy beliefs. Journal of Sport and Exercise Psychology, 27(2), 135-151. https://doi.org/10.1123/jsep.27.2.135

Schunk, D. H., \& Carbonari, J. P. (1984). Self-efficacy models. Behavioral health: A handbook of health enhancement and disease prevention, 230-247.

Schwarzer, R. (2008). Modeling health behavior change: How to predict and modify the adoption and maintenance of health behaviors. Applied Psychology, 57(1), 1-29. https://doi.org/10.1111/j.1464-0597.2007.00325.x

Schwarzer, R. (2014). Self-efficacy in the adoption and maintenance of health behaviours: Theoretical approaches and a new model. Self-efficacy: Thought control of action: Taylor \& Francis.

Schwarzer, R., \& Chung, R. (1996). Anticipating stress in the community: Worries about the future of Hong Kong. Anxiety, stress, and coping, 9(2), 163-178. http://dx.doi.org/10.1080/10615809608249399

Schwarzer, R., Luszczynska, A., Ziegelmann, J. P., Scholz, U., \& Lippke, S. (2008). Social-cognitive predictors of physical exercise adherence: three longitudinal studies in rehabilitation. Health Psychology, 27(1S), 54-63. http://psycnet.apa.org/doi/10.1037/0278-6133.27.1(Suppl.).S54

Sharma, S. V., Hoelscher, D. M., Kelder, S. H., Diamond, P., Day, R. S., \& Hergenroeder, A. (2010). Psychosocial factors influencing calcium intake and bone quality in middle school girls. Journal of the American Dietetic Association, 110(6), 932-936. https://doi.org/10.1016/j.jada.2010.03.013

Sherwood, N. E., \& Jeffery, R. W. (2000). The behavioral determinants of exercise: implications for physical activity interventions. Annual review of nutrition, 20(1), 21-44. https://doi.org/10.1146/annurev.nutr.20.1.21

Strathdee, S. A., Mausbach, B., Lozada, R., Staines-Orozco, H., Semple, S. J., Abramovitz, D., . . . Martínez-Mendizábal, G. (2009). Predictors of sexual risk reduction among Mexican female sex workers enrolled in a behavioral intervention study. Journal of acquired immune deficiency syndromes (1999), 51(Suppl 1), S42. https://dx.doi.org/10.1097\%2FQAI.0b013e3181a265b2

Strauss, R. S., Rodzilsky, D., Burack, G., \& Colin, M. (2001). Psychosocial correlates of physical activity in healthy children. Archives of Pediatrics \& Adolescent Medicine, 155(8), 897-902. https://doi.org/10.1001/archpedi.155.8.897

Strecher, V. J. (1983). A minimal-contact smoking cessation program in a health care setting. Public Health Reports, 98(5), 497.

Strecher, V. J., McEvoy DeVellis, B., Becker, M. H., \& Rosenstock, I. M. (1986). The role of self-efficacy in achieving health behavior change. Health education quarterly, 13(1), $73-92$. https://doi.org/10.1177/109019818601300108

Therawiwat, M., Fungladda, W., Kaewkungwal, J., Imamee, N., \& Steckler, A. (2005). Community-based approach for prevention and control of dengue hemorrhagic fever in Kanchanaburi Province, Thailand. http://imsear.hellis.org/handle/123456789/34555

Yehle, K. S., \& Plake, K. S. (2010). Self-efficacy and educational interventions in heart failure: a review of the $\begin{array}{llll}\text { literature. } \quad \text { Journal } & \text { Cordiovascular } & \text { Nursing, } & \text { 25(3), }\end{array}$ https://doi.org/10.1097/JCN.0b013e3181c71e8e 


\section{Copyrights}

Copyright for this article is retained by the author(s), with first publication rights granted to the journal.

This is an open-access article distributed under the terms and conditions of the Creative Commons Attribution license (http://creativecommons.org/licenses/by/4.0/). 